\title{
UNA REVISIÓN DEL MOVIMIENTO EDUCATIVO CIENCIA-TECNOLOGÍA-SOCIEDAD
}

MEMBIELA IGLESIA, P.

Universidad de Vigo. EU Formación del Profesorado de Ourense. Vicente Risco, 9. 32001 Ourense.

\section{SUMMARY}

In this paper I present a revision of the Science-Technology-Society movement, its origins, historic development and relations with science education. Comments are made about research carried out on teacher and student opinions about this subject, and the different integration models of STS in the school curriculum. A constructivist curriculum development model is presented and questions related to content selection are considered, as well as teaching models and the conditions that curriculum materials need to fulfill to be considered as STS. Finally, a short revision of the present situation of the STS movement in Spain is made.

\section{¿CUÁL ES EL ORIGEN Y DESARROLLO HISTORICO DEL MOVIMIENTO EDUCATIVO CTS?}

EI movimiento educativo CTS surgió en los años sesenta y setenta en los campus universitarios, y se extendió a la educación secundaria en la década de los ochenta.

El movimiento CTS nace en Norteamérica como respuesta a la crisis que comenzó a aflorar a comienzos de los años sesenta en la relación que mantenía la sociedad con la ciencia y la tecnología, y que en su momento reflejaron los escritos de intelectuales tales como Snow, -al hablar de dos culturas, científica y humanista-, como Dennis Meadows -que señalaba los límites del crecimiento-, de Lewis Mumford -al comentar las consecuencias sociales de la tecnología- o Rachel Carson al llamar la atención sobre la problemática ambiental. Otros intelectuales, tales como Schumacher e Illich, introdujeron una visión crítica del impacto de la tecnología en la sociedad.

Todavía hoy en día no hay acuerdo sobre lo que significa el movimiento CTS, pero podría decirse que promueve la alfabetización científica y tecnológica de los ciudadanos para que puedan participar en el proceso democrático de toma de decisiones y en la resolucion de problemas relacionados con la ciencia y la tecnología.

\section{CTS EN LA ENSEÑANZA-APRENDIZAJE DE LAS CIENCIAS}

La importancia del enfoque CTS en la enseñanza de las ciencias experimentales ya aparece reflejado en los documentos de la ASE Alternatives for Science Education (ASE, 1979) y Education through Science (ASE, 1981) o en un documento de la NSTA (NSTA, 1982) que recomienda una formación CTS para todos los estudian. tes norteamericanos, cuantificada en un porcentaje del $5 \%$ en el nivel elemental, un $15 \%$ en Ios cursos más bajos de la secundaria y un $20 \%$ en los más altos.

El desarrollo del movimiento CTS en las ciencias experimentales se ha concretado fundamentalmente en el diseño y puesta en práctica de diversos proyectos curriculares. Entre ellos se pueden citar el ChemCom, en EEUU; el curso de nivel 11 de Science and Technology, en Canadá; el Siscon in Schools, en el Reino Unido; el PLON, en Holanda; o el Science, Technology and Society, en Australia.

Este movimiento ha adquirido tal importancia, que Yager y Roy (1993) señalaban cómo en el año 1990 había en EEUU centenares de centros de secundaria que ofrecían cursos CTS. Este enfoque ha sido reconocido como orientación para la reforma en la educación científica en diversos países del mundo (NSTA, 1993), e 
- Los conocimientos y destrezas de los profesores.

- Las cuestiones relativas al ambiente de escolarización, en aspectos tales como el tiempo y los recursos disponibles o el propio ambiente físico.

- Por último, hay que considerar el componente de los contenidos, que evidentemente no puede quedar reducido a un enfoque exclusivamente disciplinar.

La información recogida sobre estudiantes y profesores, acerca del ambiente y los contenidos son los elementos que, mediatizados por el componente teórico, determinan el diseño de los materiales. Una vez diseñados éstos, se ponen en práctica y se evalúa su efectividad para promover el aprendizaje de los estudiantes.

\section{LOS CONTENIDOS CTS}

La selección de los contenidos CTS plantea problemas similares a los que se presentan con otros tipos de contenidos y, según Hickman, Patrick y Bybee (1987), cinco serían los criterios fundamentales a considerar:

1. ¿Es directamente aplicable a la vida actual de los estudiantes?

2. ¿Es adecuado al nivel de desarrollo cognitivo y a la madurez social de los estudiantes?

3. ¿Es un tema importante en el mundo actual para los estudiantes y probablemente permanecerá como tal para una proporción significativa de ellos en su vida adulta?

4. ¿Pueden los estudiantes aplicar su conocimiento en contextos distintos de los científicos escolares?

5. ¿Es un tema por el que los estudiantes muestran interés y entusiasmo?

Los modelos de organización de los contenidos CTS son básicamente dos (Holman, 1987), pues, según cuál sea el contenido en torno al que se organiza el currículo, se habla del modelo centrado en la ciencia (caso de los proyectos curriculares Nuffield Co-ordinated Sciences o SATIS) o del modelo centrado en la aplicación o en las cuestiones sociales (caso de los proyectos curriculares The Salters' Science o CEPUP).

Según cuáles sean los problemas sociales asociados a la enseñanza CTS, se han definido dos perspectivas (Rosenthal, 1989):

- Tratar cuestiones sociales externas a la comunidad científica, tales como el calentamiento global, la guerra química o los pesticidas en los alimentos.

- Tratar cuestiones sociales internas a la comunidad científica, los denominados estudios sociales de la ciencia, donde es la propia ciencia el objeto de estudio de las ciencias sociales al ocuparse de sus implicaciones filo- sóficas, sociológicas, históricas, políticas, económicas y culturales. Un ejemplo podría ser la problemática asociada a la discriminación que han sufrido las mujeres en la profesiones médicas.

Se recomienda que en el enfoque CTS no se contemplen sólo las cuestiones sociales externas a la ciencia (Aikenhead, 1990), sino también las internas tales como los aspectos filosóficos, históricos y sociológicos asociados a la propia comunidad científica.

A modo de ejemplo, se puede señalar la selección de temas CTS realizada por un grupo de especialistas en el tema (Bybee, 1987; Bybee y Mau, 1986), en la que los primeros doce temas recomendados por orden de importancia fueron: el hambre en el mundo y los recursos alimentarios, el crecimiento de la población, la calidad del aire y de la atmósfera, los recursos de aguá, la tecnologia de guerra, la salud humana y enfermedad, la escasez de energía, el uso del suelo, las substancias peligrosas, las reacciones nucleares, la extinción de plantas y animales, y los recursos minerales.

Fensham (1987) recogió las respuestas de especialistas en eđucación científica de treinta y tres estados en relación con la introducción de CTS en el currículo científico a nivel de primaria, secundaria y universidad, en relación con veintitrés temas que consideró importantes y, aunque, según el propio autor, la muestra no es representativa, todos los encuestados menos uno consideraron que en la última década se ha producido un aumento de estos temas en el currículo al menos en dos niveles educativos y que aquellos temas que parecen haber penetrado en todos los niveles son por orden relativo: uso de la energía, conservación de los recursos naturales, nutrición, salud de la poblacion, contaminación, y tecnología y medios de comunicación.

\section{LAS ESTRATEGIAS DE ENSEÑANZA- APRENDIZAJE EN CTS}

Resulta inadecuado decir que alguna estrategia de enseñanza sea exclusiva del enfoque CTS, pero se puede afirmar que la enseñanza CTS exige un repertorio de estrategias más variado que otros tipos de enseñanza (Aikenhead, 1988; Solomon, 1989). Entre los métodos de enseñanza más utilizados podemos mencionar: el trabajo en pequeños grupos, el aprendizaje cooperativo, las discusiones centradas en los estudiantes, la resolución de problemas, las simulaciones y los juegos de roles, la toma de decisiones, y el debate y las controversias.

\section{LOS MATERIALES CURRICULARES EN CIENCIA-TECNOLOGIA-SOCIEDAD}

La escasez de materiales curriculares adecuados ha sido señalado como uno de los problemas fundamentales en 
la integración del enfoque CTS en la enseñanza de las ciencias (Aikenhead, 1992; Bybee, 1991), debido a que son pocos los profesores que tienen el tiempo, la energía y los recursos necesarios para diseñar sus propios materiales. A modo de ejemplo, se pueden tener en cuenta los criterios propuestos por el equipo de un proyecto CTS (Science through Science, Technology and Society) para considerar como tal un material determinado (Waks, 1990). Éstos son:

1. Potenciar la responsabilidad, desarrollando en los estudiantes la comprensión de su papel como miembros de la sociedad, que a su vez debe integrarse en el conjunto más amplio que constituye la propia naturaleza.

2. Contemplar las influencias mutuas entre ciencia, tecnología y sociedad.

3. Promover los puntos de vista equilibrados para que los estudiantes puedan elegir conociendo las diversas opiniones, sin que el profesor tenga necesariamente que ocultar la suya propia.

4. Ejercitar a los estudiantes en la toma de decisiones y en la solución de problemas.

5. Promocionar la acción responsable, alentando a los estudiantes a comprometerse en Ia acción social, tras haber considerado sus propios valores y los efectos que pueden tener las distintas posibilidades de acción.

6. Buscar la integración, haciendo progresar a los estudiantes hacia visiones más amplias de la ciencia, Ia tecnología y la sociedad, que incluyan cuestiones éticas $y$ de valores.

7. Promover la confianza en la ciencia, en el sentido de que los estudiantes sean capaces de usarla y entenderla en un marco CTS.

\section{REFERENCIAS BIBLIOGRÁFICAS}

ACEVEDO, J.A. (1993). ¿Qué piensan los estudiantes de Ia ciencia. Un enfoque CTS. Enseñanza de las Ciencias, núm. extra, IV Congreso, pp. 11-12.

ACEVEDO, J.A. (1994). Los futuros profesores de enseñanza secundaria ante la sociología y la epistemología de las ciencias. Un enfogue CTS. Revista Interuniversitaria de Formación del Profesorado, 19, pp. 111-125.

\section{EL MOVIMIENTO CTS EN ESPAÑA}

La influencia del movimiento CTS en los diferentes niveles del sistema educativo español ha sido hasta ahora reducida.

En la universidad se puede destacar la labor realizada por el grupo INVESCIT, entre cuyas actividades caben destacar los libros publicados en la colección Nueva Ciencia de Ia editorial Anthropos y el estudio realizado por encargo del Gobierno de Navarra para la introducción de CTS en la enseñanza secundaria.

En los niveles de enseñanza no universitaria hay que señalar el proyecto Apqua (Medir, 1995), el denominado Ciencia a través de Europa (Parejo, 1995), así como el hecho de que algunos proyectos curriculares para la enseñanza de las ciencias en secundaria se sitúan de manera explícita -caso del Projecte Gaia-, o ímplicita -caso del Proyecto ACES-, en la línea CTS.

Otro hecho importante ha sido la reciente inclusión entre las materias optativas de bachillerato (Resolución de 29 de diciembre de 1992, BOE 29-01-93) de una asignatura denominada Ciencia, Tecnología y Sociedad. Esto ha iniciado un debate sobre la orientación curricular que las autoridades educativas han imprimido a esta nueva materia (Fernández, 1995) y sobre cuáles son los profesores más adecuados para impartir su docencia.

En el campo de la investigación educativa cabe destacar los trabajos realizados por Acevedo $(1993,1994)$ sobre las opiniones de estudiantes y profesores acerca de la ciencia y Ios científicos o sobre la formación del profesorado (Acevedo, 1996); los llevados a cabo por Solbes y García (1993), Solbes y otros (1993), Solbes y Vilches (1989a, 1989b, 1992, 1993, 1995) sobre diversos aspectos de la temática CTS; así como la tesis doctoral de Vilches (1993).

En el futuro, y tal como ha ocurrido en otros países, parece que asistiremos a un aumento del interés que suscita el movimiento CTS en la enseñanza-aprendizaje de las ciencias experimentales.

ACEVEDO, J.A. (1996). La formación del profesorado de enseñanza secundaria y la educación CTS. Una cuestión polémica. Revista Interuniversitaria de Formación del Profesorado, 26, pp. 131-144.

AIKENHEAD, G.S. (1984). Teacher decision making: The case of Prairie High. Journal of Research in Science Teaching, Vol. 21 , pp. $167-186$. 
AIKFNIIEAD, G.S. (1987). High-school graduates 'beliefs about science-technology-society. IIX. The characteristics and limitations of scientific knowledge. Science Education, $71(2)$, pp. 459-487.

AIKFNHEAD, G.S. (1988). An analysis of Four Ways of Assessing Student Beliefs about STS Topics. Journal of Research in Science Teaching, 25(8), pp. 607-629

AIKFNHEAD, G.S. (1990). Consequences to learning science through STS: A research perspective. Comunicación presentada al curso del British Council sobre Science, Technology and Society Education. Londres: British Council.

AIKFNHEAD, G.S. (1992). The Integration of STS into Science Education. Theory into Practice, $31(1)$, pp. 27-35.

AIKENHEAD, G.S. y RYAN, A.G.(1989). The development of a multiple-choice instrument for monitoring views on sciencetechnology-society topics. Otawa: Social Sciences and Humanities Research Council of Canada.

ASE (1979). Alternatives for Science Education. Hartfield, Gran Bretaña: ASE.

ASE(1981). Education through Science. Hartfield, Gran Brctaña: ASE.

BRUNKHORST, B.J. (1987). A comparison of student/teacher positions on selected STS topics: A preliminary study, pp. 613-622, en Riquarts, K. (ed.). Science \& Technology Education and the Quality of Life, 3. Kiel, Alemania: IPN.

BYBliN, R.W. (ed.) (1986). Science, Technology, Society. NSTA Year book 1985. Washington: NSTA.

BYBLE, R.W. (1987). Teaching about science-technologysocicty (STS): Views of science educators in the United States. Science School and Mathematics, 87(4), pp. 274-285.

BYBEE, R.W. (1991). Science-Technology-Society in Science Curriculum: The Police-Practice Gap. Theory into Practice, 30(4), pp. 294-302.

BYBEE, R.W. y MAU, T. (1986). Scjence and Technology Related Global Problems. International Survey of Science Fducators. Journal of Research Science Teaching, 23(7), pp. 599-618.

CHEEK, D.W. (1992). Thinking Constructively About Science, Technology, and Society Education. Nueva York: State University of New York Press.

DRIVLR, R. y OLDHAM, V. (1986). A constructivist approach to curriculum development in science. Studies in Science Education, 13, pp. 105-122.

HERNÁNDEZ, L. (1995). La asignatura de CTS en el nuevo bachillerato. Alambique, 3, pp. 61-68.

FENSHAM, P.J. (1987). Changing to a science, society and technology approach, en Lewis, J.L. y Kelly, P.J. (eds.). Science and Technology Education and Future Human Needs. Nueva York: Pergamon Press.

FI.FMING, R.W. (1986a). Adolescent reasoning in socioscicntific issues. Part I: Social cognition. Journal of Science Teaching, 23(8), pp. 677-688.

FLEMING, R.W. (1986b). Adolescent reasoning in socioscientific issues. Part II: Non social cognition. Journal of Science Teaching, 23(8), pp. 689-698.

FLEMING, R.W. (1990). Teacher's views of technology. Final report. Saskatoon, Canadá: University of Saskatchewan.
HICKMAN, F.M., PATRICK, J.J, y BYBEE, R.W. (1987). Science / technology/society: A framework for curriculum reform in secondary school science and social studies. Colorado, EEUU: Social Science Education Consortium.

HOLMAN, J.S. (1987). Resources or courses? Contrasting approaches to the introduction of industry and technology to the secondary curriculum. School Science Review, 68 , pp. 432-438.

JAMES, R.K. (ed.) (1986). Science, Technology and Society: Resources for Science Educators. 1985. Columbus, EEUU: AETS Yearbook. AETS y SMEAC.

MEDIR, M. (1995). El proyecto APQUA. Alumbique, 3, pp. $53-60$.

MITClIENER, P. y ANDERSON, R.D. (1989). Developing and Implementing an STS Curricuitum. Journal of Research in Science Teaching, 26(4), pp. 351-369.

NSTA (1982). Science-technology-society: Science education for the 1980 s. Washington: NS'TA.

NSTA (1993). Science/Technology/Society: A New Lffort for Providing Appropiate Science for All, pp. 3-5, en Yager, R.E. (ed.), The Science, Technology, Society Movement. What Research Says to the Science Teacher, Vol.7. Washington: NSTA.

PAREJO, C. (1995). El proyecto ciencia a través de Europa. Alambique, 3, pp. 45-52.

ROSENTHAL, D.B. (1989). Two approaches to STS education. Science Education, 73(5), pp. 581-589.

RUBBA, P.A. (1989). An investigation of the semantic meaning assigned to concepts affiliated with STS education and of STS intructional practices among a sample of exemplary science teachers. Journal of Research in Science Teaching, 26(8), pp. 687.702

RUBBA, P.A. (1991). Integrating STS Into School Science and Teacher Education: Beyond Awareness. Theory into Pratice, 30(4), pp. 303-315.

RUBBA, P.A. y WIESENMAYER, R.L. (1988). Goals and Competencies for Precollege STS Education: Recommendations Based upon Recent Literature in Environmental Education. Journal of Environmental Education, 19(4), pp. 38-44.

RYAN, A.G. (1987). High School graduates'beliefs about STS. The characteristics of scientists. Science Education, $71(4)$, pp. 489-510.

SHAMOS, M.H. (1995). The myth of scientific literacy. New Brunswick, EEUU: Rutgers University Pres.

SOLbES, J. y GARCÍA, A.R. (1993). Propuesta de diseño curricular de física y química en $F S O$ desde una perspectiva CTS. Enseñanza de las Ciencias, núm. extra, IV Congreso, pp. 287-288.

SOLBES, J., NEBOT, V, y RIBELLES, R. (1993). Una actividad CTS en las ciencias de la naturaleza de $4^{\circ}$ de USO: l'hort de trenor. Enseñanza de las Ciencias, núm. extra, IV Congreso, pp. 131-132.

SOLBES, J. y VILCHES, A. (1989a). Interacciones ciencia/ fécnica, entorno natural y social: una propuesta de materiales. Enseñanza de las Ciencias, núm. extra, III Congreso (Tomo 2), pp. 302-306.

SOLBES, J. y VILCHES, A. (1989b). Interacciones cienciatécnica-sociedad: Un instrumento de cambio actitudinal. Enseñanza de las Ciencias, 7(1), pp. 14-20. 
SOLBES, J. y VILCHES, A. (1992). El modelo constructivista y las relaciones ciencia-tecnología-sociedad (CTS). Enseñanza de las Ciencias, 10(2), pp. 181-186.

SOLBES, J. y VILCHES, A. (1993). El modelo de enseñanza por investigación y las relaciones CTS. Resultados de una experiencia llevada a cabo con alumnos de BUP y COU. Enseñanza de las Ciencias, núm. extra, IV Congreso, pp. 133-134.

SOLBES, J. y VILCHES, A. (1995). El profesorado y las actividades CTS. Alambique, 3, pp. 30-38.

SOLOMON, J. (1988). The Dilemma of Science, Technology and Society Education, pp. 266-281, en Fensham, P. (ed.) Development and Dilemmas in Science Education. Londres: The Falmer Press.

SOLOMON, J. (1989). The social construction of school science, pp. 126-136, en Millar, R. (ed.), Doing science: Images of Science in Science Education. Nueva York: The Falmer Press.

SOLOMON, J. (1993). Teaching Science, Technology and Society. Filadelfia, EEUU: Open University Press.

SOLOMON, J. y AlKENHEAD, G.S. (1994). STS Education: International Perspectives of Reform. Nueva York: Teachers College Press.

SPECTOR, B.S. (1986). Inservice Teacher Preparation in STS: Perspective and Program, pp. 65-9I, en James, R.K. (ed.). Science, Technology and Society: Resources for Science
Educators. AETS Yearbook 1985. Columbus, EEUU: AETS y SMEAC.

VILCHES, A. (1993). Las interacciones CTS y la enseñanza de las ciencias fisicoquímicas. Tesis doctoral. Universidad de Valencia.

WAKS, L. (1990). Educación en ciencia, tecnología y sociedad: orígenes, desartollos internacionales y desafíos actuales. pp. 42-75, en Medina, M. y Sanmartín, J. (eds.). Ciencia, tecnologia y sociedad. Estudios interdisciplinares en la universidad, en la educación y en la gestión pública. Barcelona: Anthropos.

YAGER, R.E (ed.) (1992). The Status of Science-TechnologySociety Reform Efforts around the World. ICASE Yearbook 1992. Petersfield, Gran Bretaña: ICASE.

YAGER, R.E. (ed.) (1993). The Science, Technology, Society Movement. What Research Says to the Science Teacher, 7. Washington: NSTA.

YAGER, R.E. (1996). Science/Technology/Society as Reform in Science Education. Albany, EEUU: Suny Press.

YAGER, R.E. y ROY, R. (1993). STS: Most Pervasive and Most Radical of Reform Approaches to Science Education, pp. 7.13, en Yager, R.E. (ed.). The Science, Technology, Society Movement. What Research Says to the Science Teacher, 7. Waskington: NSTA.

ZIMAN, J. (1980). Teaching and learning about science and society. Cambridge, Gran Bretaña: Cambridge University Press.

[Artículo recibido en abri] de 1995 y aceptado en noviembre de 1996.] 
